\title{
Role of a TRIM72 ADP-ribosylation cycle in myocardial injury and membrane repair
}

\author{
Hiroko Ishiwata-Endo, ${ }^{1}$ Jiro Kato, ${ }^{1}$ Akihiko Tonouchi, ${ }^{1}$ Youn Wook Chung, ${ }^{1}$ Junhui Sun, ${ }^{2}$ \\ Linda A. Stevens, ${ }^{1}$ Jianfeng Zhu, ${ }^{1}$ Angel M. Aponte, ${ }^{3}$ Danielle A. Springer, ${ }^{4}$ Hong San, ${ }^{5}$ \\ Kazuyo Takeda, ${ }^{6}$ Zu-Xi Yu, ${ }^{6}$ Victoria Hoffmann, ${ }^{7}$ Elizabeth Murphy, ${ }^{2}$ and Joel Moss ${ }^{1}$ \\ ${ }^{1}$ Pulmonary Branch, ${ }^{2}$ Cardiovascular Branch, ${ }^{3}$ Proteomics Core Facility, ${ }^{4}$ Murine Phenotyping Core, ${ }^{5}$ Animal Surgery \\ and Resources Core, and ${ }^{6}$ Pathology Core, National Heart, Lung, and Blood Institute, NIH, Bethesda, Maryland, USA. \\ 'Diagnostic and Research Service Branch, Division of Veterinary Resources, NIH, Bethesda, Maryland, USA.
}

\begin{abstract}
Mono-ADP-ribosylation of an (arginine) protein catalyzed by ADP-ribosyltransferase 1 (ART1) - i.e., transfer of ADP-ribose from NAD to arginine - is reversed by ADP-ribosylarginine hydrolase 1 (ARH1) cleavage of the ADP-ribose-arginine bond. ARH1-deficient mice developed cardiomyopathy with myocardial fibrosis, decreased myocardial function under dobutamine stress, and increased susceptibility to ischemia/reperfusion injury. The membrane repair protein TRIM72 was identified as a substrate for ART1 and ARH1; ADP-ribosylated TRIM72 levels were greater in ARH1-deficient mice following ischemia/reperfusion injury. To understand better the role of TRIM72 and ADP-ribosylation, we used C2C12 myocytes. ARH1 knockdown in C2C12 myocytes increased ADP-ribosylation of TRIM72 and delayed wound healing in a scratch assay. Mutant TRIM72 (R207K, R260K) that is not ADP-ribosylated interfered with assembly of TRIM72 repair complexes at a site of laser-induced injury. The regulatory enzymes ART1 and ARH1 and their substrate TRIM72 were found in multiple complexes, which were coimmunoprecipitated from mouse heart lysates. In addition, the mono-ADP-ribosylation inhibitors vitamin $\mathrm{K}_{1}$ and novobiocin inhibited oligomerization of TRIM72, the mechanism by which TRIM72 is recruited to the site of injury. We propose that a mono-ADP-ribosylation cycle involving recruitment of TRIM72 and other regulatory factors to sites of membrane damage is critical for membrane repair and wound healing following myocardial injury.
\end{abstract}

Conflict of interest: The authors have declared that no conflict of interest exists.

License: Copyright 2018, American Society for Clinical Investigation.

Submitted: October 17, 2017

Accepted: October 11, 2018

Published: November 15, 2018

Reference information: JCI Insight. 2018;3(22):e97898 https://doi.org/10.1172/jici. insight.97898.

\section{Introduction}

Mono-ADP-ribosylation is a posttranslational protein modification, in which ADP-ribose is transferred from NAD to an acceptor amino acid. It was first identified as a mechanism of disease pathogenesis in the bacterial diseases such as cholera, diphtheria, and pertussis, where toxins ADP-ribosylate critical regulatory and biosynthetic proteins (1-3). Mammalian tissues have enzymatic activities that mimic those of the bacterial toxins (4-6). Mammalian arginine-specific mono-ADP-ribosylation, however, is a reversible modification of protein. Arginine-specific mono-ADP-ribosyltransferases (ARTs) (e.g., ART1, ART5), transfer ADP-ribose from NAD to arginine residues of target proteins, and ADP-ribosylarginine hydrolase 1 (ARH1) reverses the reaction by cleaving the ADP-ribose-(arginine) protein bond (7-9). Data are consistent with ART and ARH1 serving as opposing arms of an arginine ADP-ribosylation cycle.

Mono-ADP-ribosylated proteins identified in mammalian cells include actin and integrin $\alpha 7$ in mouse cardiac and skeletal muscle cells in vitro (10), and FGF-2 in a human hepatoma cell line (11). ADP-ribosylated human neutrophil peptide-1 was identified in vivo in human airways, in patients with asthma and pulmonary fibrosis (12). In the last 4 years, significant advances have led to the identification of mono-ADP-ribosylated proteins. Reanalysis of phosphoproteomic data identified 79 mono-ADP-ribosylated proteins, including a muscle-specific membrane repair protein, tripartite motif 72 (TRIM72), also known as Mitsugumin 53 (MG53), which was ADP-ribosylated on arginines 207 and $260(13,14)$. Another method involved enrichment of ADP-ribose acceptor proteins using the ADP-ribose-binding macrodomain protein Af1521 (15-17). Similar to other posttranslational modifications, e.g., phosphorylation, mono-ADP-ribosylation can alter protein function. Potential roles for mono-ADP-ribosylation have been postulated in diabetes (18), as well as in inflammation (19) and cancer (20,21). 
TRIM72 is abundant in heart and skeletal muscle $(22,23)$ and in lung and kidney epithelial cells, where it is involved in membrane repair (24-26). Effective membrane repair protects cardiomyocytes from ischemic damage. Oligomerization of TRIM72 was required acutely for membrane repair, bringing TRIM72 to the site of injury $(22,27,28)$. Nitrosylation of TRIM72 protected it from degradation under $\mathrm{H}_{2} \mathrm{O}_{2}$-induced oxidative stress $(29,30)$. TRIM72 also countered cell damage due to ischemia/reperfusion (I/R) injury. TRIM72 in complex with caveolin-3 (Cav-3) activated the PI3K-dependent reperfusion injury salvage kinase (RISK) pathway, thereby enhancing cell survival (29-32). Overexpression of TRIM72 protected cells from plasma membrane damage in muscular dystrophy (33), as well as lung, kidney, and heart injury (25, 26, 34, 35), suggesting that TRIM72 could be a therapeutic target in diseases involving cell membrane injury.

Here, we describe cardiac fibrosis, cardiomyopathy, and reduced ejection fraction in ARH1-KO mice. We hypothesized that a mono-ADP-ribosylated protein serving as an ART1 and ARH1 substrate was involved in preserving cardiac function. We observed in ARH1-KO mouse heart in vivo, and in the Langendorff perfused-heart model ex vivo, that the amount of mono-ADP-ribosylated TRIM72 was increased in I/R injury. In cultured C2C12 myocytes, interrupting the TRIM72 mono-ADP-ribosylation cycle by knockdown of ART1, ARH1, or TRIM72 delayed membrane repair and wound healing. Overexpressing mutant TRIM72 that lacks 2 primary ADP-ribosylation sites (R207K, R260K) had a similar effect. Both ARH1 and ART1 were identified with TRIM72 in putative repair complexes. In addition, the mono-ADP-ribosylation cycle was essential for oligomerization of TRIM72 at the sites of injury. We propose that TRIM72 is ADP-ribosylated during the membrane repair process in cardiomyocytes and that proper regulation of cyclic TRIM72 ADP-ribosylation is fundamental to this critical cell repair function.

\section{Results}

Cardiomyopathy associated with myocardial fibrosis in ARH1-KO mice. ARH1-deficient (ARH1-KO) mice had reduced myocardial contractility compared with their WT counterparts, as assessed by echocardiography in M-mode (Figure 1, A and B; original images in Supplemental Material) and B-mode (Supplemental Videos 1 and 2; supplemental material available online with this article; https://doi.org/10.1172/jci. insight.97898DS1) or by MRI (Supplemental Videos 3 and 4). Evidence of cardiomyopathy was seen in representative histological sections, with left ventricular enlargement and wall thickening (Figure 1D). Myocardial fibrosis was present in $10.4 \%$ of 8 -month-old ARH1-KO mice, which was associated with increased deposition of collagen in the cardiac interstitial space (Figure 1, F and $\mathrm{H}$ ). The frequency of cardiomyopathy with cardiac fibrosis in ARH1-KO mice (22.2\%, 48 of 216 males; $2.2 \%, 7$ of 313 females) was significantly greater than that in WT $(0.4 \%, 1$ of 243 males; $0.8 \%, 2$ of 247 females $)$ or heterozygous $(0.8 \%, 2$ of 254 males; $0.5 \%, 2$ of 399 females) mice (Figure 1I). Sex difference in phenotype was significant in ARH1-KO mice, with frequency of cardiac fibrosis in male mice $\sim 10$ times that in females (Figure 1I).

Response to dobutamine-induced stress reduced in ARH1-KO mice. The dobutamine stress test (dobutamine, 0 , 10 , and $40 \mu \mathrm{g} / \mathrm{kg} / \mathrm{min}$ ) was administered to confirm that ADP-ribosylation is involved in cardiac function. Eight-month-old mice that had not developed cardiomyopathy and myocardial fibrosis were used for the dobutamine stress test. Evaluation of myocardial fibrosis was performed by histopathology on sections with H\&E stain to confirm that mice did not have myocardial fibrosis. Under basal conditions, left ventricular internal dimension (LVID) at diastole (LVIDd) in ARH1-KO male but not female mice was significantly greater than that in WT mice (Figure 1N). Myocardial contractility was increased in a dobutamine dose-dependent manner regardless of genotype or sex (Figure 1, J-U). During high-dose dobutamine ( $40 \mu 1 / \mathrm{kg} / \mathrm{min}$ ) infusion, ejection fraction (\%EF) (Figure 1, J and $\mathrm{K}$ ) and fractional shortening (\%FS) (Figure 1, L and $\mathrm{M}$ ) in ARH1-KO mice were significantly lower than those observed in WT mice, with a similar response in both sexes. During highdose dobutamine infusion, LVIDd (Figure 1, N and O) and LVID at systole (LVIDs) (Figure 1, P and Q) were significantly greater in ARH1-KO than in WT mice. Further, stroke volume (SV) was greater in ARH1-KO than in WT mice (Figure 1, R and S) regardless of sex. There was, however, no significant difference in mean heart rates between $A R H 1-\mathrm{KO}$ and WT mice (Figure 1, T and $\mathrm{U}$ ), suggesting that increased cardiac output was primarily attributable to increased SV. These findings are consistent with systolic dysfunction in ARH1-KO mice.

ADP-ribosylated TRIM72 is an ARH1 substrate. ADP-ribosylarginine content of hearts was quantified using trichloroacetic acid-fixed (TCA-fixed) tissues, as described by Jacobson et al. (36). ADP-ribosylarginine content of $A R H 1-\mathrm{KO}$ murine hearts was greater than that of WT or heterozygous mice (Figure 2A); ADP-ribosyltransferase activity did not differ among genotypes (Figure 2B), suggesting that ARH1 deficiency led to the increased ADP-ribosylarginine content of ARH1 substrate proteins. 
A
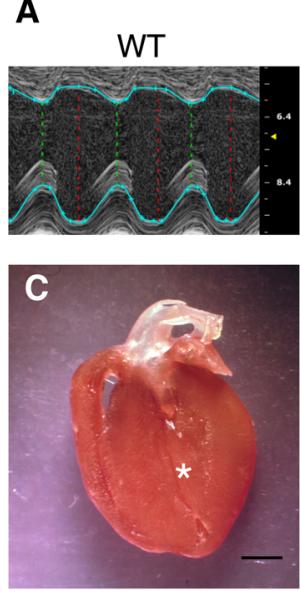

B
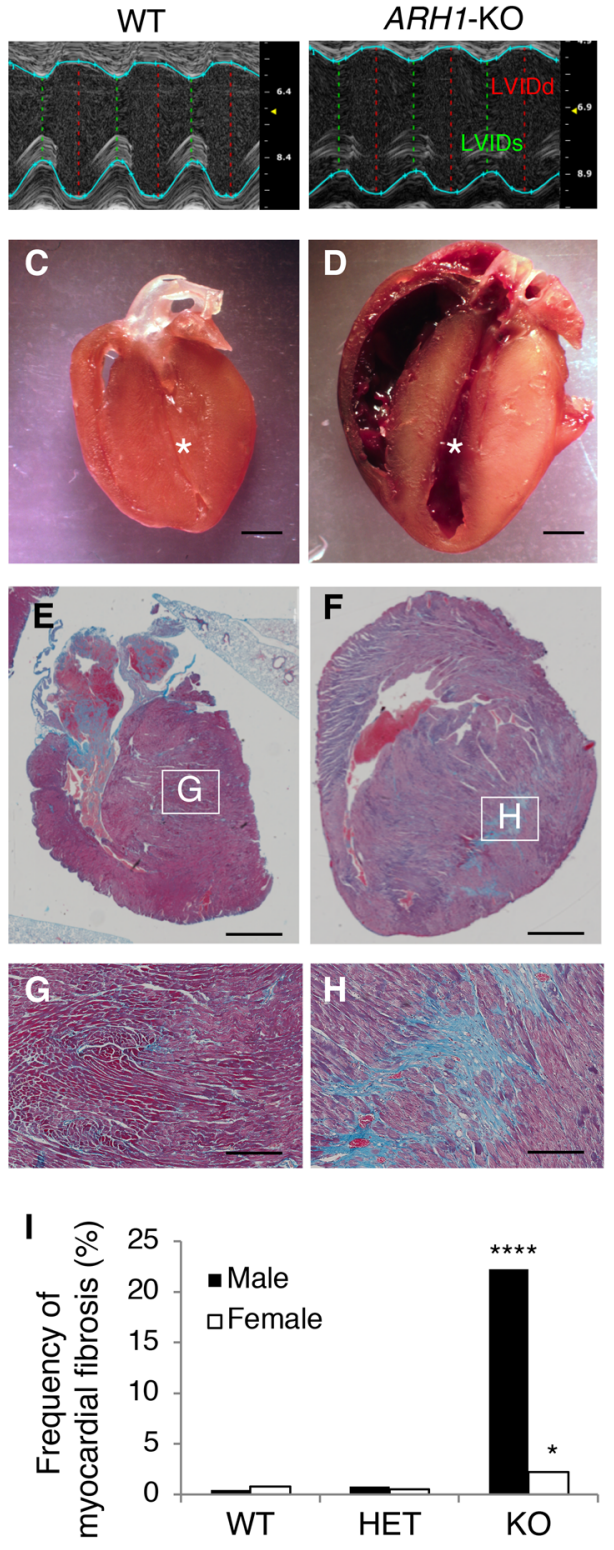
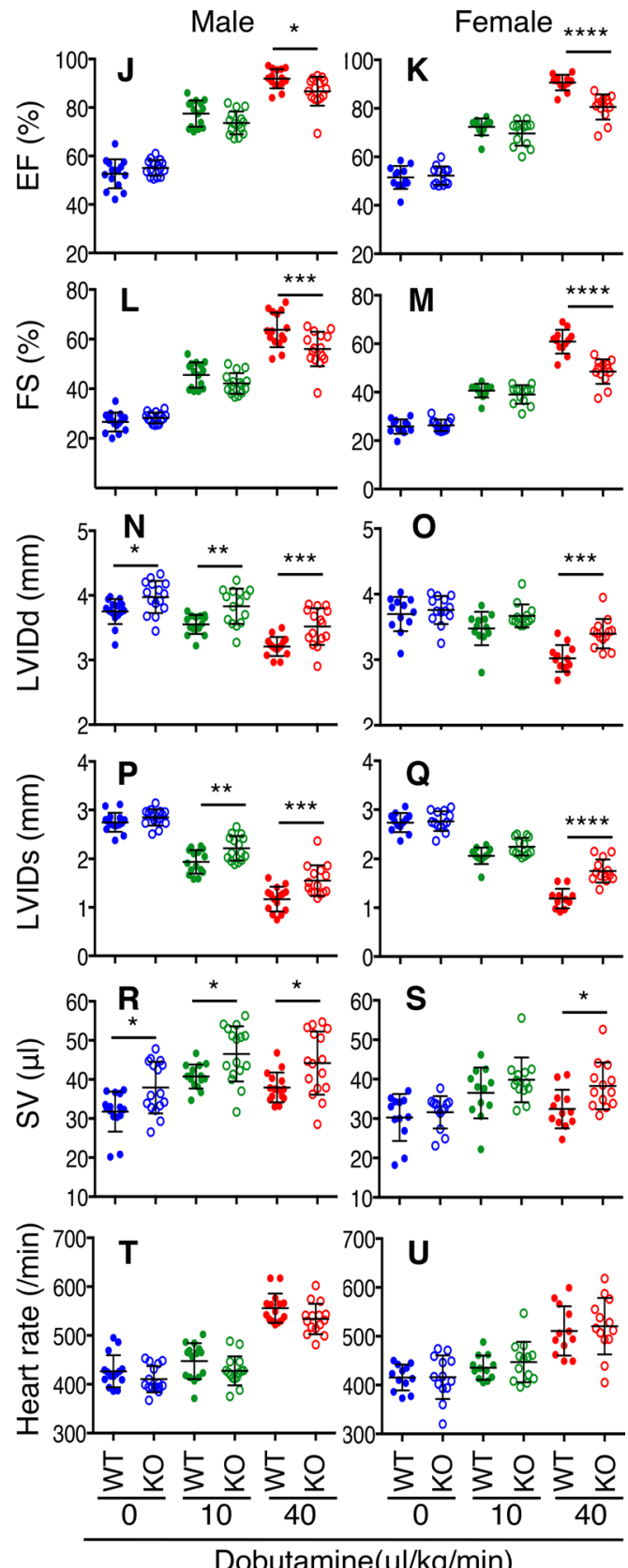

Figure 1. Impaired cardiac function with myocardial fibrosis in ARH1-KO mice. (A and B) Representative images of animals treated as described in Methods by echocardiography in M-mode from healthy WT (A) and cardiomyopathy ARH1-KO (B) mice (see also Supplemental Videos 1-4, and original images in the Supplemental Material). (C and $\mathbf{D})$ Pictures of cross section of healthy WT mouse heart (C) and 8-month-old ARH1-KO heart with cardiomyopathy (D). White asterisks indicate left ventricle (scale bars: 1 $\mathrm{mm}$ ). (E-H) Masson's trichrome staining of myocardium from WT (E and G) and $A R H 1-K O$ (F and $\mathbf{H}$ ) mice (scale bars: $200 \mu \mathrm{m})$. Histochemical staining for fibrosis shows collagen deposition in blue. (I) Frequency of myocardial fibrosis in WT (0.4\%, 1 of 243 males; $0.8 \%, 2$ of 247 females), heterozygous (HET, 0.8\%, 2 of 254 males; $0.5 \%, 2$ of 399 females) and ARH1-KO (22.2\%, 48 of 216 males; $2.2 \%, 7$ of 313 females) mice. ${ }^{*} P<0.05$, ${ }^{* * *} P<0.0001$, significant difference compared with WT of same sex by 1-sample $t$ test. (J-U) Dobutamine stress test was performed in WT males, $n=15$, $37.1 \pm 0.9$ weeks; KO males, $n=15,38.3 \pm 3.9$ weeks; WT females, $n=12$, $39.2 \pm 5.6$ weeks; KO females, $n=13,38.8 \pm 3.5$ weeks. Measurements of ejection fraction (EF; J and $\mathbf{K}$ ); fractional shortening (FS; $\mathbf{L}$ and $\mathbf{M}$ ), left ventricular internal dimension at systole (LVIDs; $\mathbf{N}$ and $\mathbf{0}$ ) and diastole (LVIDd; $\mathbf{P}$ and $\mathbf{Q}$ ), stroke volume (SV; $\mathbf{R}$ and $\mathbf{S}$ ), and heart rate ( $\mathbf{T}$ and $\mathbf{U})$. Data are shown as mean \pm SEM. ${ }^{*} P<0.05,{ }^{* *} P<0.01,{ }^{* * *} P<$ $0.001,{ }^{* * *} P<0.0001$ vs. WT of same sex by 2-way ANOVA, followed by Bonferroni's post hoc tests.

To explore whether cardiomyopathy in ARH1-KO mice could result from defective regulation of an ADP-ribosylated protein, proteomic analysis was used to identify proteins containing ADP-ribosylated arginine. TRIM72 was identified as an ARH1 target protein by 2-dimensional fluorescence difference gel electrophoresis (2D-DIGE), followed by liquid chromatography-tandem MS (LC-MS/MS). Green spots (Figure 2C, 2D-DIGE) indicate ADP-ribosylated proteins that are in excess in the ARH1-deficient tissues. ADP-ribosylarginine hydrolase activity (Figure 2C, PBS and ARH1) and chemical sensitivity (Figure 2C, NaCl and $\mathrm{NH}_{2} \mathrm{OH}$ ) demonstrated that $\mathrm{ARH} 1$ and $\mathrm{NH}_{2} \mathrm{OH}$ removed ADP-ribose from TRIM72 ADP-ribosylated on arginine, resulting in an alkaline shift of the TRIM72 isoelectric point. As expected, ADP-ribosylated TRIM72 was bound by Af1521, an ADP-ribose-binding macrodomain protein (17), and binding was inhibited by free ADP-ribose. The inactive Af1521 macrodomain mutant did not bind ADP-ribosylated TRIM72 (Figure 2D).

ADP-ribosylation of TRIM72 was enhanced by I/R injury. Localization of TRIM72 to injury sites where it provides protection against cardiac I/R injury has been reported (32). We therefore tested the hypothesis that ARH1 activity has a role in TRIM72-mediated membrane protection during myocardial infarction 
A

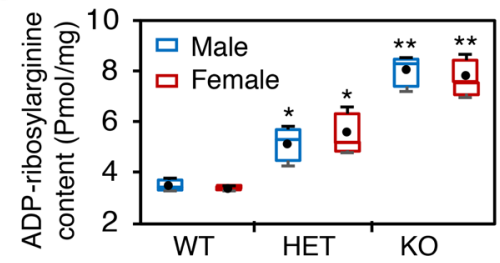

B
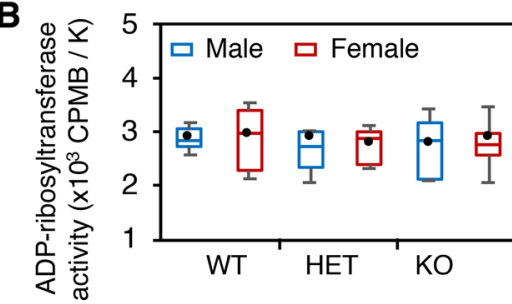

D

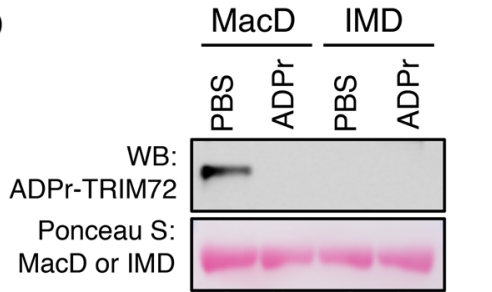

C

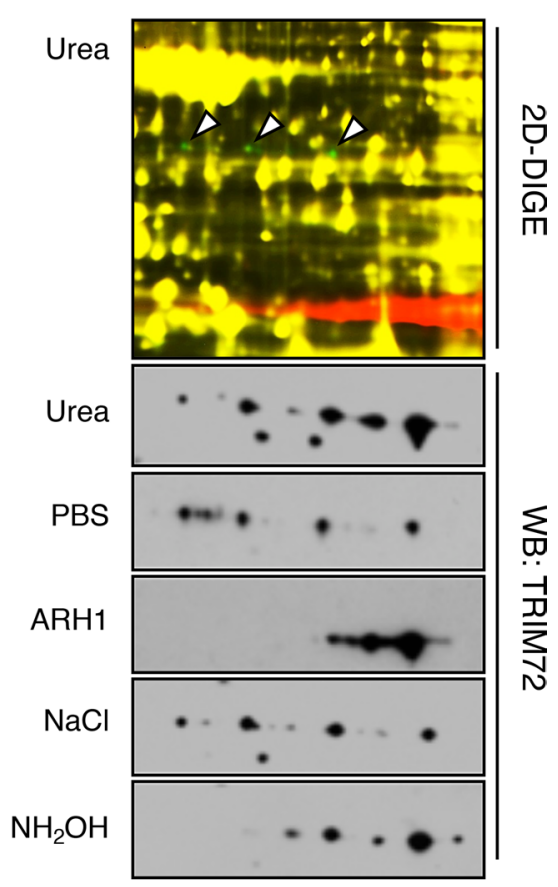

Figure 2. Identification of ADP-ribosylated TRIM72 as an ARH1 substrate in mouse heart. (A) ADP-ribosylarginine content of ARH1-deficient (KO), heterozygous (HET), and WT mouse hearts. (B) Cardiac ADP-ribosyltransferase activity of ARH1-KO, HET and WT mice. (C) Identification of ADP-ribosylated TRIM72 in ARH1-KO mouse hearts. Two-dimensional fluorescence difference gel electrophoresis (2D-DICE) image. Heart lysates from ARH1-KO mice were incubated with recombinant mouse ARH1 (Cy5, red) or PBS (Cy3, green). Two-dimensional gel separation, followed by Western blot (WB) images show TRIM72 status after treatment with urea, PBS, ARH1, NaCl, and $\mathrm{NH}_{2} \mathrm{OH}$. (D) Active Af1521 macrodomain-CST (MacD), but not inactive Af1521 macrodomain-GST (IMD), bound to ADP-ribose (ADPr) on TRIM72. Af1521 macrodomain-CST $(0.5 \mu \mathrm{mol} / 100 \mu \mathrm{l})$ or inactive Af1521 macrodomain-GST $(0.5 \mu \mathrm{mol} / 100 \mu \mathrm{l})$ were preincubated for 1 hour at $4^{\circ} \mathrm{C}$ with ADP-ribose $(1 \mu \mathrm{mol} / 100 \mu \mathrm{l})$ or PBS, then incubated with TCA-treated (Supplemental Figure 2) mouse heart lysates $\left(500 \mu \mathrm{g} /\right.$ reaction) overnight at $4^{\circ} \mathrm{C}$ in a pull-down assay to concentrate ADP-ribosylated TRIM72 (ADPr-TRIM72), which then was identified by immunoreactivity using Western blots with anti-TRIM72 antibody. Boxand-whisker plots show median, first and third quartiles (boxes), and minimum and maximum values (whiskers). $n=3$ in each group; ${ }^{*} P<0.05,{ }^{* *} P<0.01$ vs. WT of same sex by 1-way ANOVA and Bonferroni's post hoc test. Representative images of 3 or 4 independent experiments are shown.

(MI). Induced I/R injury was greater in a Langendorff isolated perfused-heart model in ARH1-KO (75.7\% $\pm 7.9 \%, n=11)$ than in WT $(37.6 \% \pm 6.1 \%, n=9)$ hearts (Figure 3C). Consistent with this finding, postischemic rate pressure product (RPP) of $A R H 1$-KO hearts $(22.1 \% \pm 5.9 \%, n=11)$ was significantly lower than that of the WT hearts $(40.7 \pm 3.6 \%, n=9)$ at 90 minutes of reperfusion (Figure 3B). In addition, ischemic preconditioning (IPC) failed to protect $A R H 1$-KO hearts, although it did decrease myocardial injury and improved RPP of WT hearts (Figure 3, B and C). Af1521 macrodomain-GST coimmunoprecipitation confirmed that ADP-ribosylated TRIM72 levels were higher in ARH1-KO than WT myocardium (Figure 3D). ADP-ribosylation of TRIM72 was enhanced by I/R injury, but not by ischemia only, suggesting that cardiac membrane protection during I/R could depend on ADP-ribosylation of TRIM72 (Figure 3D). MI in vivo reproduced the I/R injury seen ex vivo, which was significantly greater in vivo in ARH1-KO (38.3 $\pm 2.0 \%, n=7)$ than WT mice $(18.1 \% \pm 1.7 \%, n=7$ ) (Figure 3F). ADP-ribosylation of TRIM72 (Figure $3 \mathrm{G}$ ) and disruption of TRIM72 localization on membrane (Supplemental Figure 3) were enhanced by I/R, but not by ischemia only. There was no difference in left ventricular pressure between $A R H 1$-KO and WT mice (Table 1), suggesting that the differences in I/R-induced infarction were driven by effects on cell membrane viability, but not on blood pressure. Our data are consistent with the hypothesis that ARH1 plays an important role in regulating cardiac membrane protection/repair, through events dependent on TRIM72 ADP-ribosylation.

TRIM72 is an ART1 substrate. Our data showed that ADP-ribosylated TRIM72 was an ARH1 substrate, which was present intracellularly, whereas arginine-specific ARTs had been identified on the cell surface 
A

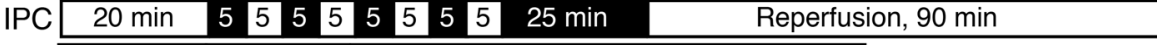

\begin{tabular}{|c|c|c|}
\hline$1 / \mathrm { R } \longdiv { \overline { 2 0 \mathrm { min } } }$ & $25 \mathrm{~min}$ & Reperfusion, $90 \mathrm{~min}$ \\
\hline
\end{tabular}

IS 20 min 25 min

B

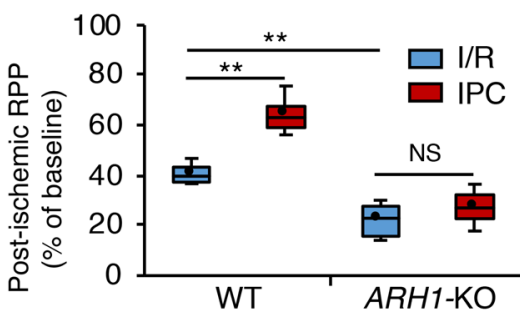

C

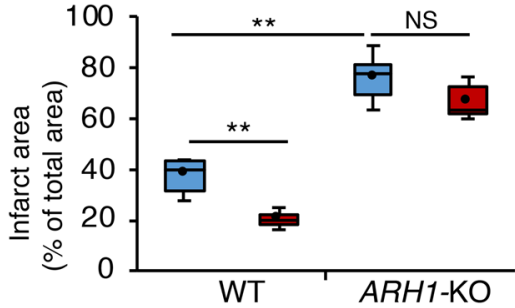

$\mathbf{F}$

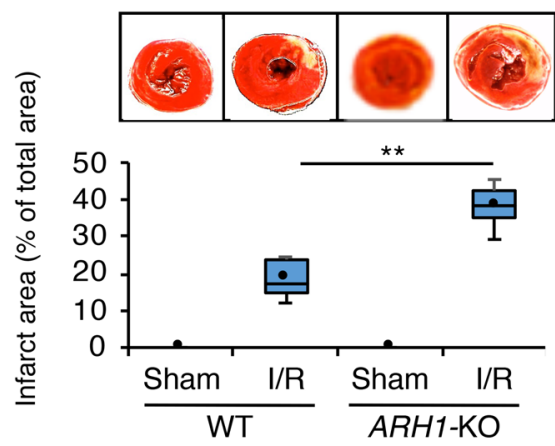

D $\quad \frac{\text { Control }}{\mathrm{WT} K O} \frac{\mathrm{IS}}{\mathrm{WT} K O} \frac{\mathrm{I} / \mathrm{R}}{\mathrm{WT} K O} \frac{\mathrm{IPC}}{\mathrm{WT} \mathrm{KO}}$

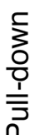

$m$

$\rightarrow$

WB: ADPr-TRIM72

Ponceau S:MacD

WB: ARH1

WB: TRIM72

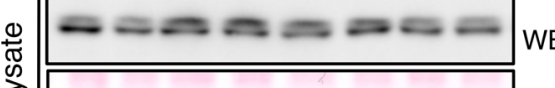

ponceau S

Input (1/5)

E is $2 \mathrm{~h}$

$2 \mathrm{~h}$

$2 \mathrm{~h}$

Ischemia

G

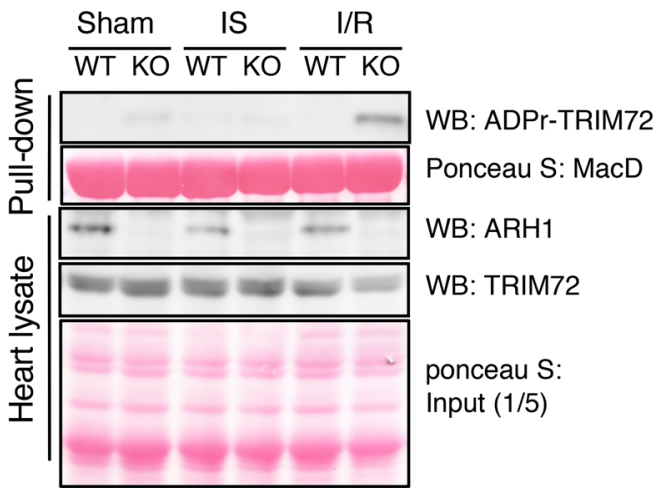

Figure 3. Ischemia/reperfusion injury enhanced ADP-ribosylation of TRIM72 in ARH1-KO mouse heart in vivo and in perfused heart model. (A) Langendorff isolated perfused heart model protocol. IPC, ischemic preconditioning; I/R, ischemia/reperfusion; IS, ischemia. (B and C) Recovery of postischemic left ventricular (postischemic rate pressure product [RPP]) (B) and myocardial infarct size (C) after I/R or IPC in ARH1-KO mice (male, $n=6,33.9 \pm 0.8$ weeks, $n=5,34.5 \pm 1.3$ weeks; female, $n=6,34.8 \pm 0.4$ weeks, $n=5,33.9 \pm 1.9$, respectively) and in WT mice (male, $n=4,33.6 \pm 1.3$ weeks, $n=5$, $37.9 \pm 1.8$; female, $n=4,34.4 \pm 0.6$ weeks, $n=5,38.5 \pm 2.9$, respectively). (D) ADP-ribosylated TRIM72 collected by Af1521 macrodomain-CST pull-down assay ( $n=3)$. (E) Myocardial ischemia/reperfusion protocol in vivo. (F) Images of TTC stain and measurement of infarct size after sham operation and in vivo I/R in ARH1-KO ( $n=6, n=7$, respectively) and WT ( $n=7, n=7$, respectively) mice. (C) Endogenous ADP-ribosylated TRIM72 increased after I/R in ARH1-KO but not WT mice. ADP-ribosylated TRIM72 was bound to immobilized Af1521 macrodomain-CST (Af1521). ADPr-TRIM72 coimmunoprecipitated with Af1521 macrodomain-GST was detected by Western blotting (WB) with anti-TRIM72 antibody. Box-and-whisker plots show median, first and third quartiles (boxes), and minimum and maximum values (whiskers). ${ }^{* *} P<0.01$, by 1-way ANOVA, post hoc Student-Newman-Keuls test. Immunoblot data are representative of 3 experiments.

(ART1) (10) or secreted into the extracellular compartment (ART5) $(5,6)$. We observed that ART1, but not ART5, transferred $\left[{ }^{32} \mathrm{P}\right] \mathrm{ADP}$-ribose from $\left.{ }^{32} \mathrm{P}\right] \mathrm{NAD}$ to recombinant mouse TRIM72 (Figure 4A). We then monitored ART1 levels in C2C12 myocytes during myogenesis, as well as those of TRIM72; ARH1; the membrane-marker protein Cav-3; and myogenin, a myogenic marker protein. Amounts of TRIM72, Cav-3, and myogenin proteins changed in parallel, increasing during myoblast differentiation into myotubes. Similarly, ART1 and ARH1 levels increased during myogenesis (Figure 4B), in agreement with the increase in arginine-specific ART activity (Figure 4C). After subcellular fractionation of WT mouse hearts, NAD:arginine ADP-ribosyltransferase activity found in membrane $(1.62 \mathrm{nmol})$ was 11.7 times greater than in cytosolic $(0.14$ nmol) fractions (Figure 4D). ART activity was released from the surface of C2C12 myocytes (Figure 4E) by phosphatidylinositol-specific phospholipase C (PI-PLC), which cleaves glycosylphosphatidylinositol (GPI) anchors attached to proteins, including ART1 (6). 
Table 1. Hemodynamic parameters

\begin{tabular}{|l|c|c|c|c|}
\hline \multirow{2}{*}{ Parameters } & \multicolumn{2}{|c|}{ Male } & \multicolumn{2}{c|}{ Female } \\
\cline { 2 - 5 } & $\mathrm{WT}(n=8)$ & $A R H 1-\mathrm{KO}(n=8)$ & WT $(n=9)$ & $41 \pm 1$ \\
\hline Age (wk) & $42 \pm 1$ & $44 \pm 1$ & $30 \pm 1$ & $29 \pm 2$ \\
\hline Body weight (g) & $39 \pm 3$ & $42 \pm 2$ & $424 \pm 7$ & $427 \pm 16$ \\
\hline Heart rate (bpm) & $441 \pm 12$ & $452 \pm 4$ & $5.1 \pm 0.4$ & $27.3 \pm 1.1$ \\
\hline Cardiac output (ml/min) & $5.2 \pm 0.3$ & $5.2 \pm 0.3$ & $26.8 \pm 1.1$ & $112 \pm 3$ \\
\hline EF (\%) & $27.7 \pm 1.3$ & $27.2 \pm 1.5$ & $116 \pm 2$ & $3.5 \pm 0.9$ \\
\hline LV maximum pressure (mmHg) & $138 \pm 3$ & $140 \pm 4$ & $115 \pm 2$ & $111 \pm 3$ \\
\hline LV minimum pressure (mmHg) & $4.2 \pm 0.6$ & $4.2 \pm 1.4$ & $11.6 \pm 1.4$ & $11.6 \pm 1.5$ \\
\hline LVESP (mmHg) & $137 \pm 3$ & $139 \pm 4$ & $12.7 \pm 1.8$ & \\
\hline LVEDP (mmHg) & $12.5 \pm 1.5$ & $8,429 \pm 282$ & $-167 \pm 316$ & $7,324 \pm 321$ \\
\hline LV dP/dt max (mmHg/s) & $8,262 \pm 288$ & $-8,878 \pm 278$ & $-8,016 \pm 320$ & $-8,538 \pm 437$ \\
\hline LV dP/dt min (mmHg/s) & $-8,580 \pm 438$ & & \\
\hline
\end{tabular}

Data shown are mean \pm SEM analyzed by 2-way ANOVA, followed by Bonferroni's post hoc tests. $n$, number of mice; EF, ejection fraction; LV, left ventricle; LVESP, left ventricular end-systolic pressure; LVEDP, left ventricular end-diastolic pressure; LV dP/dt max, maximal rate of rise of left ventricular pressure; $\mathrm{LV} d P / d t$ min, minimal rate of rise of left ventricular pressure.

Membrane repair and cell motility during wound healing are regulated by an ART1/TRIM72/ARH1 ADP-ribosylation cycle. Potential biological functions for TRIM72 ADP-ribosylation cycle proteins were evaluated in $\mathrm{C} 2 \mathrm{C} 12$ myocytes, which contain ART1, TRIM72, and ARH1 when differentiated into myotubes and have other components, e.g., Cav-3, of a putative muscle repair system. Western blot analysis of $\mathrm{C} 2 \mathrm{C} 12$ cell lysates after transfection with TRIM72, ARH1, ART1, and ARH1 plus ART1 shRNA demonstrated decreased protein expression compared with cells transfected with control shRNA (Figure 5A). We used a laser injury model to test the role of ADP-ribosylated TRIM72 on acute membrane repair. After laser injury of C2C12 myocyte membranes, live-cell imaging revealed rapid accumulation of WT TRIM72GFP at the site, which was markedly lower in cells depleted of ARH1 or ART1. Moreover, accumulation of double mutant TRIM72(R207K,R260K)-GFP at the injury site was substantially lower than that of WT TRIM72-GFP (Figure 5, B and C, and Supplemental Videos 5-10). In wound-healing assays (Figure $5, \mathrm{D}$ and $\mathrm{E}$ ), scratch repair in 24 hours was significantly reduced after knockdown of ARH1, ART1, or TRIM72 than that seen with control cells $(96.6 \% \pm 1.0 \%)$. As seen in Figure 5, D and E, TRIM72 knockdown had a milder phenotype than ARH1 or ART1 single knockdown. Furthermore, double knockdown of ARH1 and ART1 resulted in a more severe phenotype, i.e. failure of wound closure, than in others. These results suggested the possibility of other ADP-ribosylated proteins involved in membrane repair. TRIM72 was, however, the major ADP-ribosylated protein identified in the ARH1-KO mouse heart by the 2D-DIGE system. To assess effects of TRIM72 ADP-ribosylation on wound healing, pEGFP-TRIM72-N1 or non-ADP-ribosylatable mutant pEGFP-TRIM72 (R207K, R260K)-N1, in which arginines 207 and 260 were replaced with lysines, were expressed in TRIM72-knockdown cells. The extent of wound closure at 24 hours (Figure 5, D and E) was significantly improved by expression of WT TRIM72-GFP (88.4\%) in TRIM72-knockdown cells (62.3\%), whereas expressing mutant TRIM72 (R207K, R260K) did not improve repair rate (41.9\%). These data suggest that ARH1, ART1, and TRIM72 ADP-ribosylation is required for wound healing. After multiple scratchings, levels of ADP-ribosylated TRIM72 in ARH1-depleted cells were higher than those in control cells (Figure 5F). In addition, Af1521 macrodomain-GST pulled down ADP-ribosylated TRIM72 (ADPr-TRIM72) and ADP-ribosylated TRIM72-GFP (ADPr-TRIM72-GFP), but not mutant TRIM72(R207K,R260K)-GFP from scratched cells (Figure 5F). These data confirm the importance of the TRIM72 ADP-ribosylation cycle in wound healing. TRIM72 had been described as an essential component of the membrane repair machinery, recruiting intracellular vesicles to sites of membrane disruption $(22,24)$.

TRIM72 ADP-ribosylation repair complexes contribute to membrane repair. Based on these findings, we propose that ART1 and ARH1 participate in an ADP-ribosylation-regulated, TRIM72-centered repair process and asked whether components of the cycle, ART1 and ARH1, were present in complexes with TRIM72. Anti-TRIM72 antibodies immunoprecipitated ART1, ARH1, and Cav-3 from WT hearts. Both anti-ART1 and anti-ARH1 antibodies reciprocally immunoprecipitated TRIM72 and Cav-3, as 
A

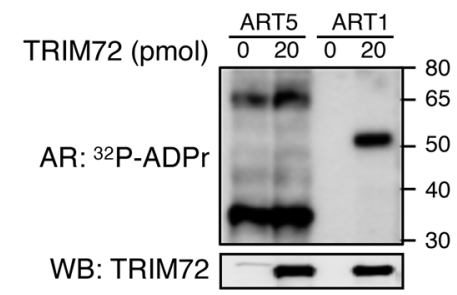

B

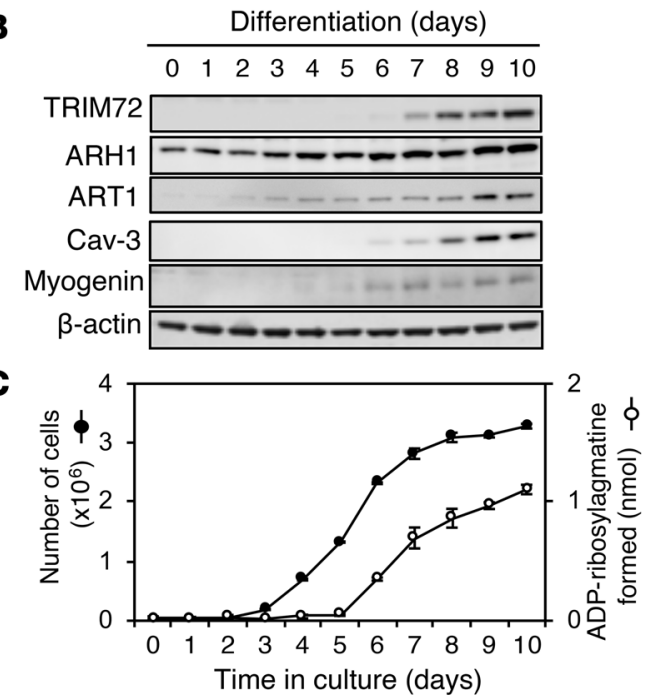

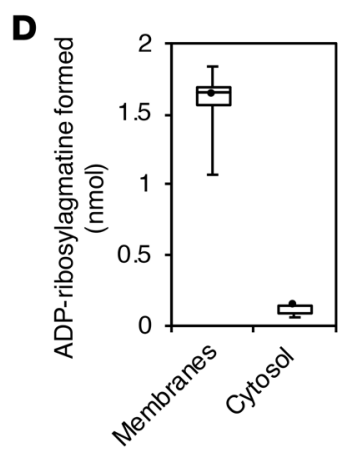

$\mathbf{E}$

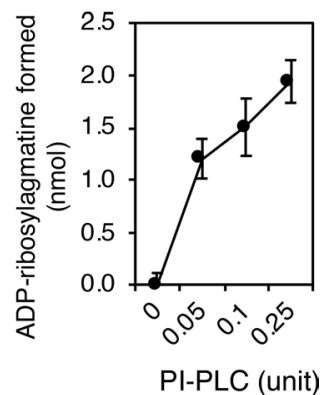

Figure 4. ADP-ribosylation of TRIM72 by ART1. (A) In ADP-ribosylation assay, recombinant murine ART1 but not ART5 $\left(0.56 \mathrm{nmol} \mathrm{NAD}\right.$ :arginine ADP-ribosyltransferase activity, respectively) transferred ${ }^{32} \mathrm{P}-\mathrm{ADP}$ ribose (32 P-ADPr) from ${ }^{32} \mathrm{P}-\mathrm{NAD}$ to recombinant mouse TRIM72. AR, autoradiography; WB, Western blot. Representative images are shown of 2 experiments in duplicate. (B) Western blot analysis of TRIM72, ARH1, ART1, caveolin-3 (Cav-3), and myogenin during C2C12 cell differentiation, with $\beta$-actin as internal control. The C2C12 cells were differentiated on day 7 expressing differentiation marker proteins, myogenin, TRIM72, and caveolin-3. Images representative of 3 experiments. (C) Number of cells (filled symbols) and ADP-ribosyltransferase activity of C2C12 cell lysates (open symbols) correlated with appearance of myogenin, TRIM72, and Cav-3 in B. Data are mean \pm SD $(n=4)$. (D) ADP-ribosyltransferase activity after subcellular fractionation of WT mouse hearts. Box-and-whisker plots show median, first and third quartiles (boxes), and minimum and maximum values (whiskers) ( $n=6$ ). (E) C2C12 myotubes were incubated with phosphatidylinositol-specific phospholipase C (PI-PLC), before assay of ADP-ribosyltransferase activity in medium released by PI-PLC. Data are mean \pm SD $(n=6)$.

well as ARH1 and ART1, respectively, from WT heart lysates, suggesting that TRIM72, ARH1, ART1, and Cav-3 are components of multimolecular complexes (Figure 6A). Furthermore, blue native PAGE separated TRIM72, ARH1, ART1, and Cav-3 complexes at about the same molecular weight (Supplemental Figure 8). This result suggested the existence of heterogeneous complexes containing TRIM72, with components of a reversible ADP-ribosylation cycle.

It has been reported that TRIM72 oligomerization serves as a key mechanism for regulation of acute membrane repair (22). Oligomerization of TRIM72, observed in WT mice, was delayed in ARH1-deficient heart lysates (Figure 6B), where a dimer of TRIM72 rather than a trimer was seen. Similarly, oligomerization of TRIM72 was blocked by vitamin $\mathrm{K}_{1}$ and novobiocin, inhibitors of arginine-specific mono-ADP-ribosylation (37) (Figure 6C). Following overexpression of TRIM72-GFP, as well as mutant TRIM72(R207K,R260K)-GFP in C2C12 cells, both WT TRIM72-GFP and mutant TRIM72(R207K,R260K)-GFP formed dimers, but not trimers. The WT heart, however, formed a TRIM72 trimer (Supplemental Figure 7). Due to this result, we did not perform further experiments regarding oligomerization using the $\mathrm{C} 2 \mathrm{C} 12$ cell model. ADP-ribosylated TRIM72 was found among the monomeric forms of TRIM72, but not TRIM72 oligomers (Figure 6D). Together, these results suggest that optimal repair required both ART and ARH1 activity. Removal of ADP-ribose from ADP-ribosylated TRIM72 by ARH1 promoted oligomerization of TRIM72, thereby promoting membrane repair (Figure 6, B-D). 
A
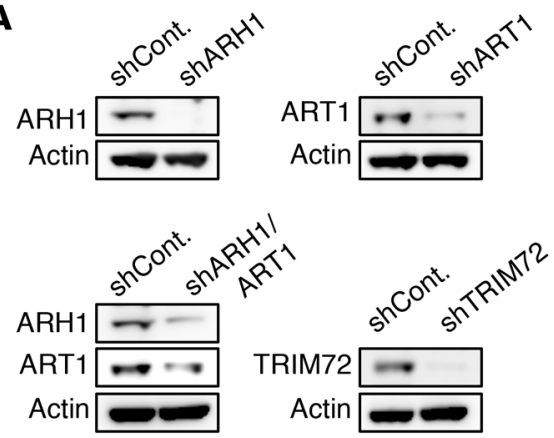

B
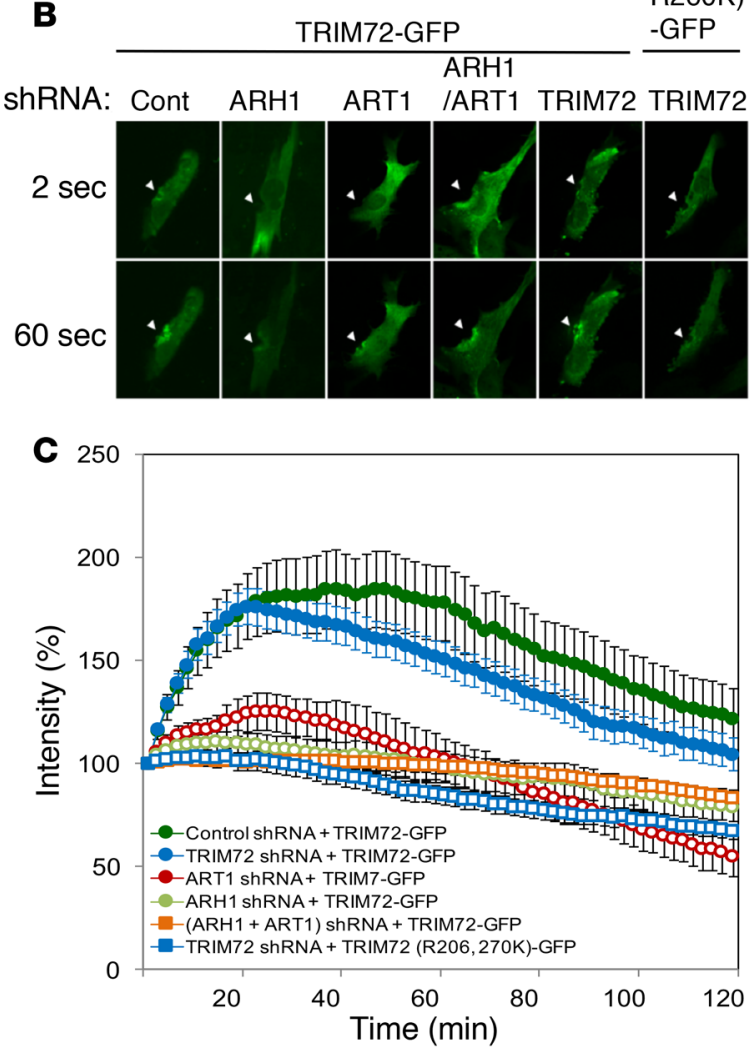

D

TRIM72

(R207K

TRIM72 R260K)

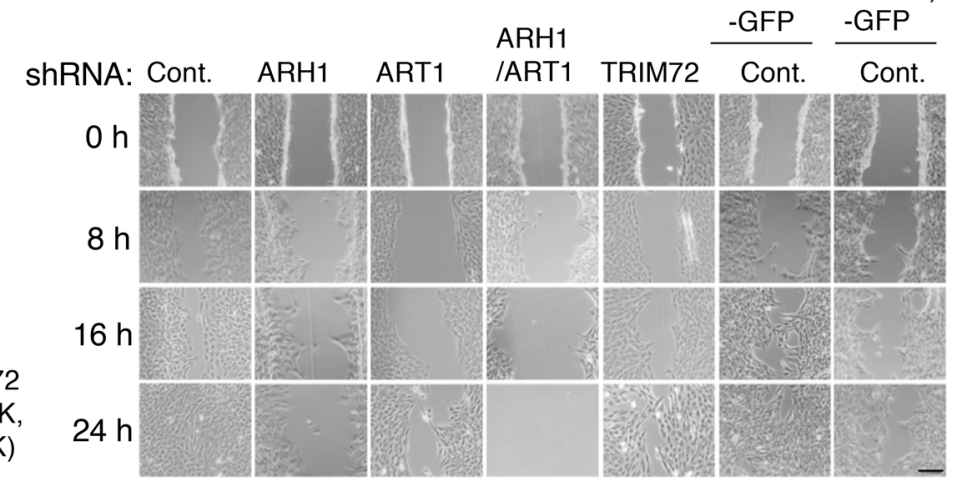

E

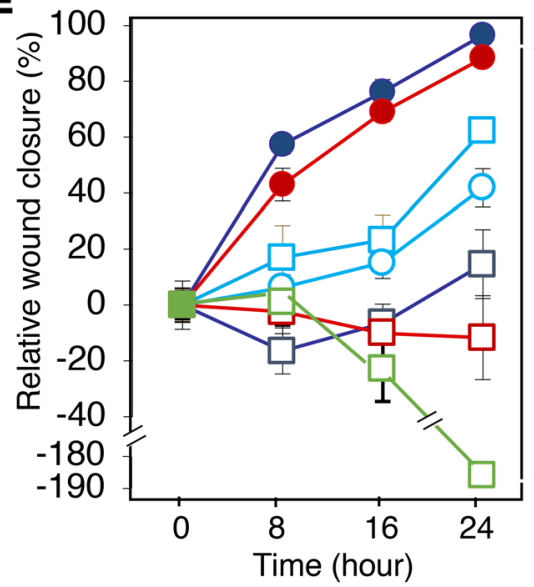

$\longrightarrow$ - shCont.

- - shCont._TRIM72-GFP

- - shTRIM72

$\longrightarrow$ shCont._TRIM72(R207K, R260K)-GFP

- - ShART1

$\longrightarrow$ shARH

- $=$ ShARH1/ART1

TRIM72

(R207K,

TRIM72 R260K)

F

ARH1/ -GFP -GFP

shRNA: Cont. ARH1 ART1 TRIM72 ART1 Cont. Cont.

Scratch: $\overline{-+} \overline{-++} \overline{-+} \overline{-+}+\overline{t+}$

WB:

ADPr-TRIM72

Ponceau S: MacD or IMD
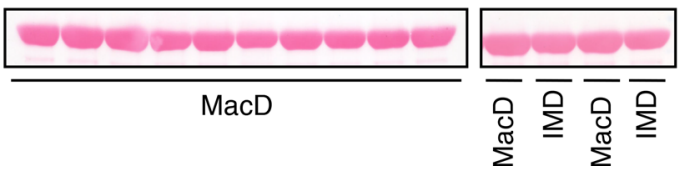

Figure 5. Depletion of ARH1, ART1, and TRIM72 in C2C12 cells delayed wound healing and membrane repair. (A) Western blot analysis of C2C12 cell lysates after TRIM72 (shTRIM72), ARH1 (shARH1), ART1 (shART1), and ARH1 plus ART1 (shARH1/ART1) shRNA transformation, demonstrating decreased protein expression compared with control scrambled shRNA (shCont.) transfectant. (B and C) Laser wounding assays were performed with C2C12 cells transformed with TRIM72-GFP in control scrambled shRNA $(n=35)$, ARH1 shRNA $(n=34)$, ART1 shRNA $(n=31)$, TRIM72 shRNA ( $n=35)$, or double knockdown for shART1 and shARH1 ( $n=31$ ) with or without overexpression with WT TRIM72-GFP or mutant TRIM72(R207K,R260K)-GFP ( $n=37$ ) (B) (Supplemental Videos 5-10). (C) Measurements of GFP intensity at injury sites. (D and E) Scratch wound healing assays were performed with cells transformed with shCont., shARH1, shART1, shTRIM72, or shART1/ARH1 knockdown cells with or without overexpression with mutant TRIM72(R207K,R260K)-GFP or WT TRIM72-GFP. Scale bar: $1 \mathrm{~mm}$. (E) Quantification of the area covered by cells at each time is shown as percentage of time 0 wound area $(n=12)$. (F) Western blots are shown for detection of ADP-ribosylated TRIM72 coimmunoprecipitated with active or inactive Af1521 macrodomain-CST from C2C12 myotube lysates with scratching with needles. The amount of ADP-ribosylated TRIM72 was increased after scratching of C2C12 cells transformed with control shRNA or ARH1 shRNA. In addition, in scratched C2C12 myocytes, Af1521 macrodomain-GST pull-down revealed that WT TRIM72-GFP, but not mutant-TRIM72(R207K,R260K)-GFP, was ADP-ribosylated. ADPr-TRIM72 coimmunoprecipitated with Af1521 macrodomain-GST was detected by Western blotting (WB) with anti-TRIM72 antibody. Data shown are representative of 3 experiments. Data are mean \pm SEM. Open symbols are values significantly different $(P<0.05)$ from those of shCont. cells by 2-way ANOVA, followed by Bonferroni's post hoc tests.

\section{Discussion}

Our study was initiated with observations in living ARH1-KO mice and moved to an ex vivo perfused heart model, then to a $\mathrm{C} 2 \mathrm{C} 12$ myocyte model, leading to demonstration of an ADP-ribosylation complex involved in membrane repair. We identified endogenous ADP-ribosylated TRIM72 following I/R-induced 
A
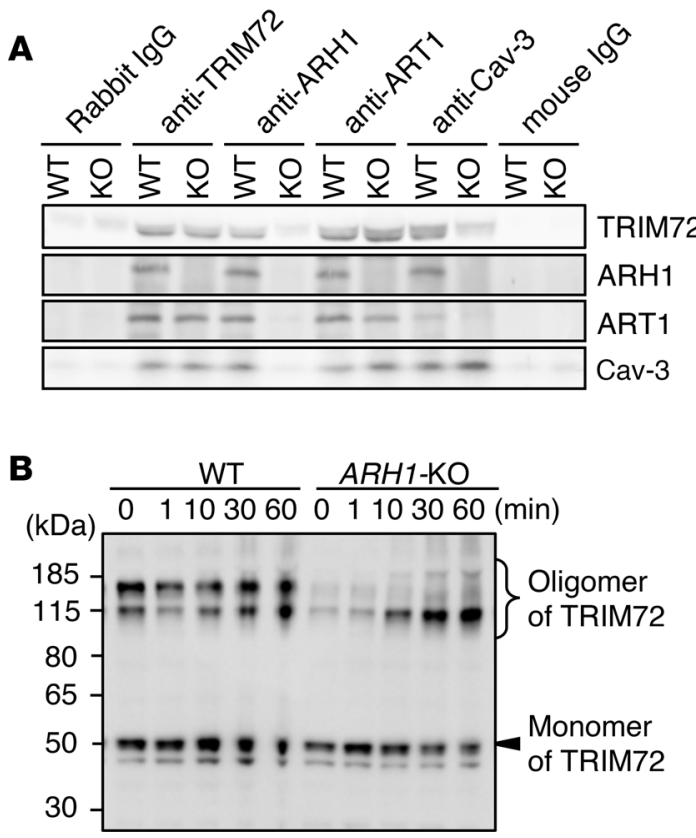

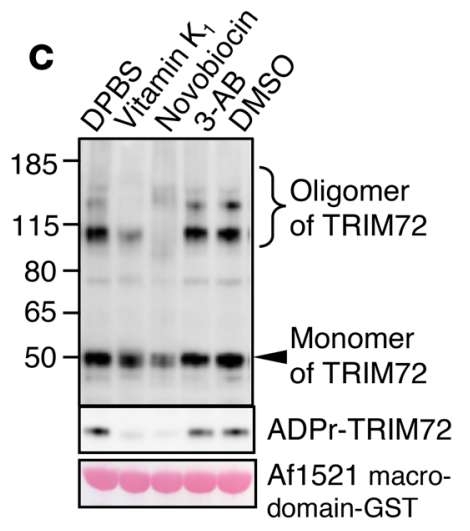

D

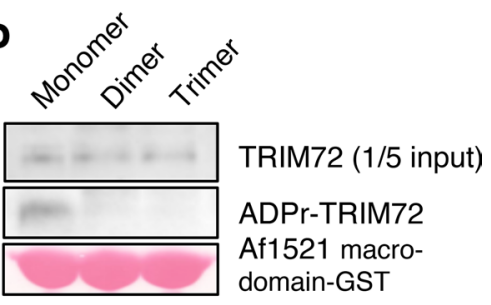

Figure 6. TRIM72 oligomerization and complex formation required the enzymatic components of the TRIM72 ADPribosylation cycle. (A) WT and ARH1-KO (KO) mouse heart lysates were subjected to immunoprecipitation with the indicated antibodies and normal IgG, followed by Western blotting with anti-TRIM72, anti-ARH1, anti-ART1, or antiCav-3 antibodies. (B) Oligomers of TRIM72 seen in WT and ARH1-KO heart lysates. (C) TRIM72 oligomerization was observed in WT mouse heat lysates treated with mono-ADP-ribosylation inhibitors, vitamin $\mathrm{K}_{1}$ or novobiocin, or the poly ADP-ribosylation inhibitor 3-aminobenzamide (3-AB). After treatment with/without inhibitor, heart lysates were subjected to pull-down assay with Af1521 macrodomain-GST. (D) TRIM72 in its monomeric form but not in oligomers was found to be ADP-ribosylated. TRIM72 in monomeric, dimeric, and trimeric forms was excised according to molecular weight. ADPr-TRIM72 coimmunoprecipitated with Af1521 macrodomain-CST was detected by Western blotting (WB) with anti-TRIM72 antibody. Data shown are representative of 3 experiments.

myocardial injury in the $A R H 1-\mathrm{KO}$ mouse (Figure 3). Using C2C12 cells, we demonstrated that a TRIM72 ADP-ribosylation cycle accelerated membrane repair at sites of injury (Figure 5). We also showed that TRIM72, ART1, ARH1, and Cav-3 were coimmunoprecipitated (Figure 6A) and detected in complexes at about the same molecular weight by blue native PAGE electrophoresis, followed by Western blot analysis (Supplemental Figure 8), consistent with their participation in macromolecular complexes that regulate the cyclic ADP-ribosylation of TRIM72 during membrane repair.

Cardiomyopathy and cardiac fibrosis were observed in ARH1-KO mice (Figure 1), consistent with a critical role for the TRIM72 ADP-ribosylation cycle in maintaining normal membrane physiology and accelerating repair. The frequency of myocardial fibrosis seen in ARH1-KO male mice was 10 times greater than in female mice (Figure 1I). Likewise, cardiomyopathy is more prevalent in men than women (38). On the other hand, the dobutamine-induced stress test showed decreased cardiac contractility in ARH1-KO mice, regardless of sex (Figure 1, J-U), perhaps suggesting a role for ARH1 in cardiac function. A genome-wide association study reported one case of cardiomyopathy in the $\mathrm{In}(7 \mathrm{Oca} 2 ; 7 \mathrm{Sox} 6) 100 \mathrm{H}$ mouse, which has chromosome 7 inversion with ART1 and ART5 gene mutations (39). This result supports a critical role of arginine-specific mono-ADP-ribosylation in cardiac function. Overall, our findings are consistent with participation of TRIM72, ART1, ARH1, and Cav-3 in macromolecular complexes that regulate membrane repair. It has been reported that a heterodimer of TRIM72 and Cav-3 was essential for membrane repair following acute muscle damage (40). In agreement, Cav-3 coimmunoprecipitated with ARH1 in our experiments (Figure 6A). Blue native PAGE is a versatile method for analysis of macromolecules in their native state $(41,42)$. Using blue native PAGE, we detected complexes containing 53-kDa TRIM72, 39-kDa ART1, $39-\mathrm{kDa}$ ARH1, and 20-kDa Cav-3 at molecular masses of between about 480 and $550 \mathrm{kDa}$ (Supplemental Figure 8), consistent with heterogeneous TRIM72-containing complexes. Taken together, the results indicate that ADP-ribosylation-dependent TRIM72 complexes may play a role in maintaining cell membranes, with loss of a functional cycle leading to cardiomyopathy with myocardial injury and myocardial fibrosis. 


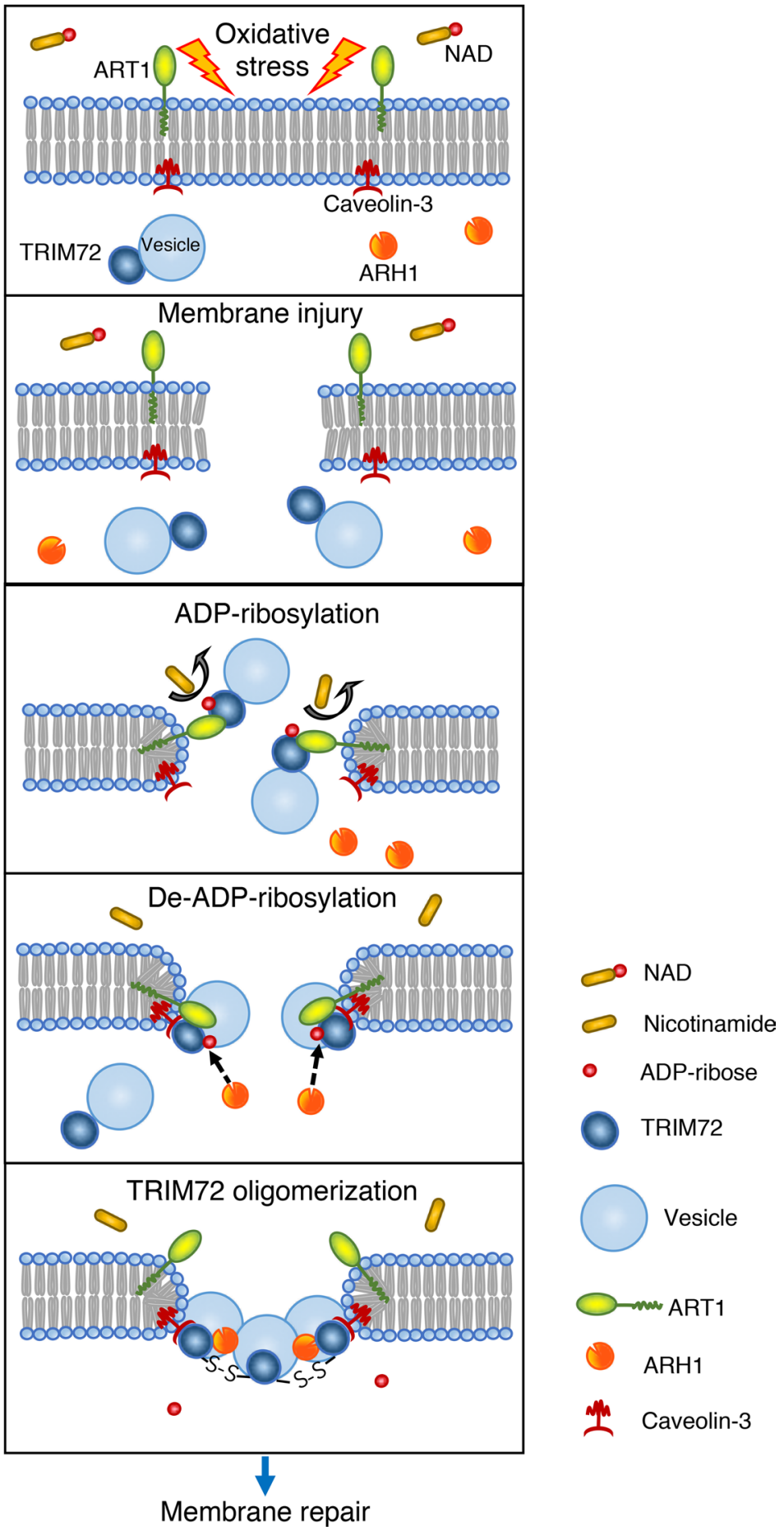

Figure 7. Schematic representation of the effects of TRIM72 ADP-ribosylation cycle on membrane repair. Ischemia/ reperfusion-induced membrane disruption results in ADP-ribosylation of TRIM72 by ART1 at the injured sites, thereby facilitating binding of TRIM72 and caveolin-3 on the membrane. It has been reported that oligomerization of TRIM72 is essential for acute membrane repair to recruit TRIM72 and intracellular vesicles toward the injury sites (21). ARH1 catalyzes de-ADP-ribosylation, cleaving the ADP-ribose from ADP-ribo sylated TRIM72; this reaction promotes oligomerization of TRIM72 at the site of injury.

Posttranslational modifications, such as $S$-nitrosylation (30) and phosphorylation (43) of TRIM72, may be important for cardiac protection. Results from our in vivo and ex vivo MI experiments (Figure 3) show that ADP-ribosylation of TRIM72 was enhanced by I/R injury, but not ischemia alone, suggesting that ADP-ribosylation of TRIM72 resulted from membrane disruption, i.e., oxidative stress, but not hypoxic stress. Under hypoxia/reoxygenation and $\mathrm{H}_{2} \mathrm{O}_{2}$-induced oxidative stress, shRNA depletion of ARH1 had a protective effect compared with control shRNA C2C12 cells (Supplemental Figure 6, A and B), which was opposite to the I/R-induced myocardial phenotype seen with the intact heart, in vivo (Figure 3, B-D). This result from isolated C2C12 cells suggested that the microenvironment of the intact heart may alter the response to mono-ADP-ribosylation.

TRIM72-KO mice were resistant to IPC (31), which involves activation of the RISK pathway, including PI3K-Akt-glycogen synthase kinase-3 $\beta$ enzymes $(44,45)$. We found that IPC did not protect $A R H 1-\mathrm{KO}$ hearts 
(Figure 3, E and F). In addition, after IPC, phospho-Akt (p-Akt) content, which appears to be protective, was lower in ARH1-KO than in WT mouse hearts (Supplemental Figure 4). In WT mice, Cav-3 formed a functional complex with TRIM72 during IPC, which activated a PI3K-Akt pathway of cardioprotection (31). We confirmed the association of TRIM72 with Cav-3 in WT mouse heart lysate, but reduced association was seen in ARH1-KO heart lysate (Figure 6A). As shown in a schematic diagram of the effects on TRIM72 ADP-ribosylation on membrane repair (Figure 7), we hypothesized that binding ADP-ribosylated TRIM72 to Cav-3 is an important step to prevent TRIM72 leakage into extracellular space and recruit TRIM72 on vesicles to the sites of injury. Likewise, ADP-ribose cleavage from ADP-ribosylated TRIM72 is a key step to stimulate TRIM72 oligomerization and promote membrane repair.

It is of practical interest to know how an extracellular protein, arginine-specific mono-ADP-ribosyltransferases (46), catalyzes ADP-ribosylation in the extracellular space where the NAD concentration is 0.1 $\mu \mathrm{M}(5,47)$. Cellular NAD is released into the extracellular matrix during inflammation and cell death (48), and TRIM72 that leaks from injured tissue into serum is a potential biomarker of injury (23). Membrane injury might also permit mixing of intracellular (e.g., NAD, ARH1, TRIM72) with extracellular factors and proteins (e.g., ART1). Consistent with these data, we hypothesize that the concentration of extracellular $\mathrm{NAD}^{+}$may increase during cardiac injury induced by I/R injury; and a membrane repair protein, such as TRIM72, may potentially be a nidus around which other components of a membrane repair system, such as those reported here, e.g., ART1, ARH1, NAD, might assemble.

In previous reports, however, other proteins, e.g., integrin $\alpha 7(48,49)$ and FGF-2 $(11)$, have been shown to be ADP-ribosylated in vitro. The only confirmed substrate of ART1 in vivo is human neutrophil peptide-1 $(12,50)$. Cholera toxin catalyzes ADP-ribosylation of Gas, a substrate of ARH1 in ARH1-KO mice in vivo (51). In this study, TRIM72 in ARH1-KO heart lysate was the major ADP-ribosylated protein that served as an ARH1 substrate (Figure 2C). Identification of ADP-ribosylated TRIM72 in WT mice has been difficult in part because of the mono-ADP-ribosylation cycle. Similarly, other reversible posttranslational modifications - e.g., phosphorylation of tyrosine, which is known to play an important role in oncogenic signaling pathways - could best be identified in the presence of tyrosine phosphatase inhibitors (52). The ARH1-KO model confirmed increased ADP-ribosylation of TRIM72 during I/R injury, where $A R H 1-\mathrm{KO}$ eliminates one arm of the cycle, showing that ADP-ribosylation of TRIM72 responds to myocardial injury (Figure 3G). Therefore, MI in vivo induced no change in ADP-ribosylated TRIM72 levels in WT mice but enhanced the cycle of mono-ADP-ribosylation consisting of the 2 enzymes, ART1 and ARH1, and TRIM72 (Figure 3G).

Oligomerization of TRIM72 is essential for acute membrane repair $(22,27)$. Our data demonstrate that either inhibition of ADP-ribosylation or its reversal prevented TRIM72 oligomerization (Figure 6, $\mathrm{B}$ and $\mathrm{C}$ ) and membrane repair (Figure 5, B and C) consistent with a TRIM72 ADP-ribosylation cycle. TRIM72 binds phosphatidylserine and cholesterol $(22,35,53)$ and is present in lipid rafts, as are other components of TRIM72 complexes, including Cav-3, a component of caveolae, and the GPI-anchored protein ART1 $(6,54)$. An intracellular protein, ARH1, was present in multimolecular complexes with Cav-3 and TRIM72 (Figure 6A). Thus, we propose that the mono-ADP-ribosylation cycle of TRIM72 results from admixture of extracellular and intracellular factors resulting from membrane damage.

\section{Methods}

Detailed descriptions of MRI, 2-dimensional gel electrophoresis and analysis, purification of recombinant proteins, design of shRNA-resistant cDNA, subcellular fractionation, cell lines, immunoprecipitation, and antibodies are provided in Supplemental Methods.

Animals. Generation of $A R H 1-\mathrm{KO}$ mice was described by Kato et al. (51). ARH1-KO mice were backcrossed 11 times with 129S6/SvEvTac mice (Taconic), and genotypes were confirmed by PCR.

Measurement of left ventricular function. A $1 \mathrm{~F}$ Millar PV catheter was used to obtain pressure-volume data in the left ventricle. The hardware, PowerLab 8/30 and MPVS-300, and software, LabChart 7, were purchased from ADInstruments.

Histology. Cardiac fibrosis was evaluated using tissue cross-sections stained with H\&E or Masson's trichrome stain. Collagen-rich fibrotic regions were stained by Masson's trichrome stain. Representative images of ARH1-KO and WT mice at 8-12 months of age are shown (Figure 1, C-H).

Dobutamine stress echocardiography. The dobutamine stress test was performed by an investigator blinded to mouse genotype. Mice were lightly anesthetized with isoflurane during examination and placed supine 
on a heated platform with electrocardiography (ECG) leads and a rectal temperature probe. Heart images were acquired using the Vevo2100 ultrasound system (VisualSonics) with a 30-MHz ultrasound probe (VisualSonics, MS-400 transducer). After baseline scan, mice were given a constant rate infusion of dobutamine $(0.625 \mathrm{mg} / \mathrm{ml}$ in normal saline containing $5 \%$ dextrose $)$ via the tail vein using an infusion syringe pump (Harvard Apparatus). The first infusion was at the low dose of $10 \mu \mathrm{g} / \mathrm{kg} / \mathrm{min}$. After the heart rate reached a steady state within $500 \mathrm{bpm}$ as determined by the ECG, infusion rate was increased to $40 \mu \mathrm{g} / \mathrm{kg}$ / min, and the scans were repeated. After the dobutamine stress test, evaluation of myocardial fibrosis was performed by histopathology on sections with $H \& E$ stain.

Pull-down ADP-ribosylated TRIM72 with Af1521 macrodomain-GST. Af1521 macrodomain-GST (0.5 $\mu \mathrm{mol} / 100 \mu \mathrm{l})$ or inactive Af1521 macrodomain-GST $(0.5 \mu \mathrm{mol} / 100 \mu \mathrm{l})$ was preincubated for 1 hour at $4^{\circ} \mathrm{C}$ with ADP-ribose $(1 \mu \mathrm{mol} / 100 \mu \mathrm{l})$ or PBS and incubated with TCA-treated (Supplemental Figure 2) mouse heart lysates (500 $\mu \mathrm{g} /$ reaction) overnight at $4^{\circ} \mathrm{C}$ in a pull-down assay to concentrate ADP-ribosylated TRIM72, which then was identified by immunoreactivity using Western blots (Figure 2D). Representative images of 3 experiments are shown. To detect ADP-ribosylation of TRIM72 following cardiac infarction, mouse hearts were immediately frozen in liquid nitrogen and ground in a mortar. After evaporation of liquid nitrogen, $8 \%$ ice-cold TCA was added. TCA-precipitated proteins, which do not have ADP-ribosyltransferase activity, were dissolved in $4 \mathrm{ml}$ ice-cold Tris- $\mathrm{HCl}$ buffer $(20 \mathrm{mM}$ Tris- $\mathrm{HCl}, \mathrm{pH}$ 7.5, $20 \mathrm{mM} \mathrm{NaCl}$ ) (Figurer 3, D and G, and Supplemental Figures 2 and 4). Protein was quantified by spectrophotometer (NanoDrop, Thermo Fisher Scientific). For pull-down of ADP-ribosylated TRIM72 with Af1521 macrodomain-GST resin (Tulip BioLabs), heart lysates were incubated with $20 \mu \mathrm{g}$ Af1521 macrodomain-GST resin with a rotator (20 reversals $/ \mathrm{min})$ overnight at $4^{\circ} \mathrm{C}$, and washed 3 times. Proteins were separated by SDS-PAGE using 10\% Bis-Tris NuPAGE gels (Invitrogen) or $10 \%$ polyacrylamide gels $(200 \times 200 \times 1.5 \mathrm{~mm}$, Hoffer SE600), and transferred to nitrocellulose membranes. Blotted membranes were blocked with TBS-T (Tris-buffered saline, $0.1 \%$ Tween-20) with $5 \%$ skim milk (Bio-Rad) at room temperature (RT) for 1 hour, then reacted with rabbit anti-TRIM72 antibody (1:1,000 dilution) overnight at $4^{\circ} \mathrm{C}$. After washing with TBS-T at RT for 10 minutes, 3 times, membranes were reacted with HRP-conjugated anti-rabbit IgG (1:5,000 dilution, Promega) at RT for 1 hour. Washing was performed as above. Enhanced chemiluminescence substrate (Immobilon Western by Millipore or SuperSignal West Pico by Pierce) was used for visualization of immunocomplexes, which were detected with Fujifilm Las-3000.

ADP-ribosylarginine hydrolysis and hydroxylamine sensitivity assays. To quantify ADP-ribosylarginine hydrolysis, heart lysate (150 $\mu \mathrm{g}$ protein/reaction) in RIPA buffer with EDTA-free protease inhibitor cocktail (Roche) was incubated $\left(2\right.$ hours, $30^{\circ} \mathrm{C}$ ) with $30 \mu \mathrm{g}$ recombinant murine ARH1 (mean $\mathrm{V}_{\text {max }}, 1.285 \mu \mathrm{mol} /$ $\mathrm{min} / \mathrm{mg}$ ) or PBS as control. To test hydroxylamine sensitivity, heart lysate (150 $\mu$ g protein) in $25 \mu 1$ RIPA buffer with EDTA-free protease inhibitors was incubated $\left(1\right.$ hour, $\left.30^{\circ} \mathrm{C}\right)$ with neutralized $4 \mathrm{M} \mathrm{NH}_{2} \mathrm{OH}(25$ $\mu$, final concentration $2 \mathrm{M}$ ) or $4 \mathrm{M} \mathrm{NaCl}(25 \mu$, final concentration $2 \mathrm{M})$ as control in $\mathrm{KPO}_{4}$ buffer, $\mathrm{pH}$ 7.4, containing $10 \mathrm{mM} \mathrm{MgCl}_{2}, 5 \mathrm{mM}$ DTT (reaction volume, $50 \mu 1$ ). Reaction was stopped by addition of an equal volume of $20 \%$ TCA. After 1 hour on ice, proteins were collected by centrifugation at 20,000 $g$ for 30 minutes for 2-dimensional electrophoresis (2DE)-Western blot analysis.

$A D P$-ribosyltransferase assays. Using procedures described previously $(55,56)$, samples of mouse heart or C2C12 cell lysates $(150 \mu \mathrm{g}$ protein/150 $\mu \mathrm{l})$ were incubated with $0.1 \mu$ mol of [adenine $-{ }^{14} \mathrm{C}$ ]NAD $(0.025 \mu \mathrm{Ci})(\mathrm{NEC} 848050 \mathrm{UC}$, PerkinElmer), $0.1 \mathrm{mM} \beta$-NAD and $20 \mathrm{mM}$ agmatine in Tris- $\mathrm{HCl}$ buffer, $\mathrm{pH} 7.4$, for 1 hour at $30^{\circ} \mathrm{C}$ before transfer to AG1-X2 (Bio-Rad) columns $(0.4 \times 4 \mathrm{~cm})$ and elution of $\left[{ }^{14} \mathrm{C}\right]$-ADP-ribose-agmatine with $5 \mathrm{ml}$ water for liquid scintillation counting (Tri-Carb Liquid Scintillation Counter, PerkinElmer).

Langendorff heart perfusion procedures. Mice were anesthetized with intraperitoneal injection of sodium pentobarbital $(50 \mathrm{mg} / \mathrm{kg})$ and anticoagulated with $50 \mu \mathrm{l}$ of heparin $(1,000 \mathrm{U})$ administration into the inferior vena cava. Hearts were quickly excised and placed in ice-cold Krebs-Henseleit buffer (KH buffer) containing $120 \mathrm{mM} \mathrm{NaCl}, 11 \mathrm{mM}$ D-glucose, $25 \mathrm{mM} \mathrm{NaHCO}_{3}, 1.75 \mathrm{mM} \mathrm{CaCl}_{2}, 4.7 \mathrm{mM} \mathrm{KCl}, 1.2 \mathrm{mM} \mathrm{MgSO}_{4}$, and 1.2 $\mathrm{mM} \mathrm{KH}_{2} \mathrm{PO}_{4}$. Aorta was cannulated on a Langendorff apparatus, and the heart perfused in retrograde fashion with $\mathrm{KH}$ buffer, oxygenated $\left(95 \% \mathrm{O}_{2} / 5 \% \mathrm{CO}_{2}\right)$, and maintained at $\mathrm{pH} 7.4$, at a constant pressure of 100 $\mathrm{cm} \mathrm{H}_{2} \mathrm{O}$ at $37^{\circ} \mathrm{C}$. After 20 minutes of perfusion or IPC ( 4 cycles of 5 minutes of ischemia and 5 minutes of reperfusion), isolated hearts were subjected to 25 minutes of no-flow ischemia, before 90 minutes of reperfusion. As shown in Figure 3, the 4 experimental groups were (i) control: mouse hearts were rapidly harvested; 
(ii) ischemia (IS): after 20 minutes of perfusion, hearts were subjected to 25 minutes of no-flow ischemia; (iii) I/R: hearts were perfused for 20 minutes, followed by 25 minutes of ischemia before 90 minutes of reperfusion; and (iv) IPC: hearts were perfused for 20 minutes, followed by 4 cycles of 5 minutes of ischemia and 5 minutes of reperfusion, before 25 minutes ischemia and 90 minutes of reperfusion.

Hemodynamic and histopathological effects of MI. To monitor left ventricular contractile function, a latex balloon connected to a pressure transducer was inserted into the left ventricle of Langendorff-perfused hearts. Left ventricular developed pressure (LVDP) was recorded and digitized using a PowerLab system (ADInstruments). RPP (LVDP $\times$ heart rate) was used as a measure of function. Postischemic functional recovery was expressed as percentage of preischemic RPP. For measurement of myocardial infarct size, after 90 minutes of reperfusion, the hearts were perfused with 1\% 2,3,5-triphenyltetrazolium chloride (TTC; Sigma-Aldrich) dissolved in KH buffer and incubated in 1\% TTC in KH buffer for 15 minutes at $37^{\circ} \mathrm{C}$, followed by fixation in $10 \%$ formalin. Infarct size was expressed as the percentage of whole area of cross-sectional slices of mouse hearts.

Experimental MI. Acute MI was induced in 10- to 12-month-old mice. Experimental MI was performed by a surgeon who was blinded to the mouse genotype. The mice were anesthetized with $1 \%-3 \%$ isoflurane. Through a skin incision extending from the axillary region to the xiphoid cartilage, the thorax was entered at either the 3rd or 4th intercostal space with a cautery. At one-third the length of the heart from the apex, left anterior descending artery was ligated with a 7.0 or 8.0 suture, and then the chest cavity was closed in layers. For mice undergoing sham operation, a ligature was placed in a corresponding location after thoracotomy, but not ligated. If reperfusion was planned, a slipknot was made and released after chest closure. At the designated times, mice were anesthetized, and $1 \mathrm{ml}$ of $1 \%$ potassium chloride solution was injected via inferior vena cava to cause cardiac arrest. Heart was rapidly excised and frozen in liquid nitrogen or sectioned in 2-mm slices that were stained with $1 \%$ TTC in PBS solution $\left(20\right.$ minutes, $37^{\circ} \mathrm{C}$ ) and fixed in $10 \%$ formalin for quantification of infarct area by NIH ImageJ. After TTC stain scan, tissue cross-sections were stained with H\&E.

Protein ADP-ribosylation assay. Recombinant mouse TRIM72 (20 pmol) was incubated with recombinant mouse ART1 and ART5 (0.56 nmol NAD, respectively) in PBS at pH 7.5, $62.5 \mathrm{nM}$ [adenylate- ${ }^{32} \mathrm{P}$ ]$\mathrm{NAD}\left(10 \mu \mathrm{Ci}\right.$ /assay), and $5 \mu \mathrm{M} \beta-\mathrm{NAD}$ in $25 \mu \mathrm{l}$ reaction volume at $30^{\circ} \mathrm{C}$ for 2 hours. After $20 \%$ TCA precipitation and centrifugation at 20,000 $\mathrm{g}$ for 30 minutes, the pellets were dissolved in $20 \mu \mathrm{SDS}$ sample buffer, and all the of proteins were separated by NuPAGE 4-12\% Bis-Tris gel (Invitrogen), transferred to a nitrocellulose membrane, and exposed to x-ray film (BioMax XAR, Kodak) for 13 hours at $-70^{\circ} \mathrm{C}$. Data shown are representative of 3 experiments.

Site-directed mutagenesis of TRIM72 (R207K, R260K). Using plasmid pEGFP-N1-TRIM72 as template, the mutant TRIM72 (R207K, R260K) that lacks ADP-ribose modification sites was constructed using the QuikChange Lightning Multi Site-Directed Mutagenesis Kit (Agilent Technologies) with oligonucleotide primers according to the manufacturer's protocol. The original sequences, 5'-(629)CGG-(631)-3' and 5'-(788)-AGG-(790)-3', were replaced with 5'-(629)-AAA-(631)-3' and 5'-(788)AAG-(790)-3', respectively. The newly constructed plasmid was sequenced by Macrogen USA and confirmed to be correct.

Membrane repair assay. The cell membrane was irradiated at $701 \mathrm{~nm}$ for 2 seconds using a Leica SP5 confocal microscope in 2-photon excitation mode equipped with a $\times 63$ water immersion objective and a $\mathrm{CO}_{2}$ chamber at $37^{\circ} \mathrm{C}$ in a humidified atmosphere. Images were captured at 2-second intervals for 2 minutes with Zeiss ZEN imaging software. The change in fluorescence intensity between each captured frame was calculated by MetaMorph image analysis software. For laser injury assays, C2C12 myoblasts were cultured in a collagen-coated, glass-bottom dish (P35GCOL-0-14-C, MatTek) and used at approximately $80 \%$ confluence to avoid effects of cell contact.

Scratch wound healing assay. C2C12 cells were prepared that stably expressed ARH1, ART1, TRIM72, and control shRNA vectors (Origene) and transiently overexpressed pEGFP-N1-TRIM72 and pEGFP-N1TRIM72 (R207K, R260K) vectors. The cells were scratched by needle at confluence, and wound-healing ability was assessed at $0,8,16$, and 24 hours following the scratch. Area of each wound was calculated with the Fiji image analysis program. To pull down ADP-ribosylated TRIM72 with Af1521 macrodomain-GST resin, the cells were wounded with multiple scratches using needles (JJ12010, John James) at 1.5-mm intervals and incubated for 10 minutes at $37^{\circ} \mathrm{C}$ with $5 \% \mathrm{CO}_{2}$. The cells were immediately $8 \%$ TCA precipitated before being dissolved in Tris-HCl buffer, $\mathrm{pH}$ 7.4. Cell lysates $(500 \mu \mathrm{g})$ were subjected to pull-down with $10 \mu \mathrm{g}$ Af1521 macrodomain-GST. Data shown are representative of 3 experiments. 
TRIM72 oligomerization assays. ARH1-deficient and WT mouse hearts were lysed in DPBS and incubated for the indicated time at $37^{\circ} \mathrm{C}$. The proteins $(50 \mu \mathrm{g})$ were separated under nonreducing conditions by $4-12 \%$ Bis-Tris SDS-PAGE, followed by immunoblotting with anti-TRIM72 antibody (Figure 6B). WT heart lysates in DPBS with/without inhibitors were incubated for 10 minutes at $37^{\circ} \mathrm{C}$. Novobiocin (final concentration, $1 \mathrm{mM}$ ), 3-aminobenzamide (3-AB) (final concentration, $3 \mathrm{mM}$ in $0.15 \% \mathrm{DMSO}$ ), and DMSO (final concentration, $0.15 \%$ ) were from Sigma-Aldrich. Vitamin $\mathrm{K}_{1}$ (final concentration, $1 \mathrm{mM}$ ) was from Hospira. After treatments, lysates were divided into 2 tubes for nonreducing SDSPAGE and pull-down assay with Af1521 macrodomain-GST, followed by Western blotting to determine TRIM72 oligomerization and ADP-ribosylated TRIM72 level, respectively (Figure 6C). The WT mouse heart lysates incubated for 1 hour at $37^{\circ} \mathrm{C}$ were applied to $4-12 \%$ Bis-Tris SDS-polyacrylamide gels under nonreducing conditions. The gel was cut according to protein markers corresponding to monomers and oligomers of TRIM72. Gel strips were homogenized with a mortar, followed by elution with PBS. After purification of proteins with a glass fiber-filled column $(0.4 \times 4 \mathrm{~cm})$, the proteins were applied to Af1521 macrodomain-GST resin or inactive macrodomain-GST resin for isolation of ADP-ribosylated TRIM72 (Figure 6D).

Densitometry and statistics. The LAS 3000 Bioimaging system with the image analysis software Image Gauge, version 4.0 (Fuji), was used for Western blot analysis. Comparisons between 2 groups were analyzed by a 1-sample $t$ test. For multiple groups, comparisons were first analyzed by ANOVA, followed by a Student-Newman-Keuls post hoc test or Bonferroni's post hoc test. Differences were considered significant at $P<0.05$, with $n$ indicating the number of independent experiments or number of mice.

Study approval. Experimental protocols for mouse studies (H-0172 and H-0271) were approved by the National Heart, Lung, and Blood Institute Animal Care and Use Committee.

\section{Author contributions}

HIE, JK, and JM designed and analyzed the research. JK generated ARH1-KO mice and helped set up the animal studies and the laser-induced damage membrane repair assay. ZXY and VH assisted with tissue processing and analyzed histopathological specimens. KT performed MRI and echocardiography. DAS performed dobutamine stress tests and analyzed echocardiography images. JZ measured ADP-ribosylarginine content. AT and AMA performed proteomic analysis. YWC, JS, HS, and EM performed I/R studies. LAS provided recombinant proteins and technical assistance for ADP-ribosyltransferase assays. HIE performed the experiments and analyzed the data. HIE, AMA, JK, DAS, and JM wrote the manuscript.

\section{Acknowledgments}

We thank Martha Vaughan (deceased), Rodney L. Levine, and Vincent C. Manganiello (deceased) for valuable advice and critical review of the manuscript. We appreciate the professional skills and advice of Christian A. Combs and Daniela Malide (Light Microscopy Core Facility, National Heart, Lung, and Blood Institute, NIH) regarding microscopy-related experiments. This research was supported by the Intramural Research Program of the NIH, National Heart, Lung, and Blood Institute.

Address correspondence to: Joel Moss, Building 10, Room 6D05, MSC 1590, Pulmonary Branch, National Heart, Lung, and Blood Institute, National Institutes of Health, Bethesda, Maryland, 20892-1590, USA. Phone: 301.496.1597; Email: mossj@nhlbi.nih.gov.

AT's present address is: Chiba Aoba Municipal Hospital, Chiba, Japan.

YWC's present address is: Severance Biomedical Science Institute, Yonsei University College of Medicine, Seoul, South Korea.

JZ's present address is: Siemens Healthcare Diagnostics Inc., Newark, Delaware, USA.

KT's present address is: Microscopy and Imaging Core Facility, Center for Biologics Evaluation and Research, Food and Drug Administration, Silver Spring, Maryland, USA. 
1. Honjo T, Nishizuka Y, Hayaishi O. Diphtheria toxin-dependent adenosine diphosphate ribosylation of aminoacyl transferase II and inhibition of protein synthesis. J Biol Chem. 1968;243(12):3553-3555.

2. Corda D, Di Girolamo M. Functional aspects of protein mono-ADP-ribosylation. EMBO J. 2003;22(9):1953-1958.

3. Katada T, Ui M. Direct modification of the membrane adenylate cyclase system by islet-activating protein due to ADP-ribosylation of a membrane protein. Proc Natl Acad Sci USA. 1982;79(10):3129-3133.

4. Zolkiewska A, Nightingale MS, Moss J. Molecular characterization of NAD:arginine ADP-ribosyltransferase from rabbit skeletal muscle. Proc Natl Acad Sci USA. 1992;89(23):11352-11356.

5. Okazaki IJ, Kim HJ, Moss J. Cloning and characterization of a novel membrane-associated lymphocyte NAD:arginine ADP-ribosyltransferase. J Biol Chem. 1996;271(36):22052-22057.

6. Okazaki IJ, Moss J. Glycosylphosphatidylinositol-anchored and secretory isoforms of mono-ADP-ribosyltransferases. J Biol Chem. 1998;273(37):23617-23620.

7. Moss J, et al. Molecular and immunological characterization of ADP-ribosylarginine hydrolases. J Biol Chem. 1992;267(15):10481-10488.

8. Okazaki IJ, Moss J. Mono-ADP-ribosylation: a reversible posttranslational modification of proteins. Adv Pharmacol. 1996;35:247-280

9. Takada T, Okazaki IJ, Moss J. ADP-ribosylarginine hydrolases. Mol Cell Biochem. 1994;138(1-2):119-122.

10. Zolkiewska A, Moss J. Integrin alpha 7 as substrate for a glycosylphosphatidylinositol-anchored ADP-ribosyltransferase on the surface of skeletal muscle cells. J Biol Chem. 1993;268(34):25273-25276.

11. Jones EM, Baird A. Cell-surface ADP-ribosylation of fibroblast growth factor-2 by an arginine-specific ADP-ribosyltransferase. Biochem J. 1997;323 (pt 1):173-177.

12. Paone G, et al. ADP-ribosyltransferase-specific modification of human neutrophil peptide-1. J Biol Chem. 2006;281(25):17054-17060.

13. Matic I, Ahel I, Hay RT. Reanalysis of phosphoproteomics data uncovers ADP-ribosylation sites. Nat Methods. 2012;9(8):771-772.

14. Leutert $\mathrm{M}$, et al. Proteomic characterization of the heart and skeletal muscle reveals widespread arginine ADP-ribosylation by the ARTC1 ectoenzyme. Cell Rep. 2018;24(7):1916-1929.e5.

15. Dani N, et al. Combining affinity purification by ADP-ribose-binding macro domains with mass spectrometry to define the mammalian ADP-ribosyl proteome. Proc Natl Acad Sci USA. 2009;106(11):4243-4248.

16. Vivelo CA, Leung AK. Proteomics approaches to identify mono-(ADP-ribosyl)ated and poly(ADP-ribosyl)ated proteins. Proteomics. 2015;15(2-3):203-217.

17. Karras GI, et al. The macro domain is an ADP-ribose binding module. EMBO J. 2005;24(11):1911-1920.

18. Gorio A, Donadoni ML, Finco C, Di Giulio AM. Endogenous mono-ADP-ribosylation in retina and peripheral nervous system. Effects of diabetes. Adv Exp Med Biol. 1997;419:289-295.

19. Welsby I, Hutin D, Gueydan C, Kruys V, Rongvaux A, Leo O. PARP12, an interferon-stimulated gene involved in the control of protein translation and inflammation. J Biol Chem. 2014;289(38):26642-26657.

20. Kato J, et al. ADP-ribosylarginine hydrolase regulates cell proliferation and tumorigenesis. Cancer Res. 2011;71(15):5327-5335.

21. Kato J, Vekhter D, Heath J, Zhu J, Barbieri JT, Moss J. Mutations of the functional ARH1 allele in tumors from ARH1 heterozygous mice and cells affect ARH1 catalytic activity, cell proliferation and tumorigenesis. Oncogenesis. 2015;4:e151.

22. Cai C, et al. MG53 nucleates assembly of cell membrane repair machinery. Nat Cell Biol. 2009;11(1):56-64.

23. Weisleder N, et al. Recombinant MG53 protein modulates therapeutic cell membrane repair in treatment of muscular dystrophy. Sci Transl Med. 2012;4(139):139ra85.

24. Jia Y, et al. Treatment of acute lung injury by targeting MG53-mediated cell membrane repair. Nat Commun. 2014;5:4387.

25. Kim SC, et al. TRIM72 is required for effective repair of alveolar epithelial cell wounding. Am J Physiol Lung Cell Mol Physiol. 2014;307(6):L449-L459.

26. Duann P, et al. MG53-mediated cell membrane repair protects against acute kidney injury. Sci Transl Med. 2015;7(279):279ra36.

27. Hwang M, Ko JK, Weisleder N, Takeshima H, Ma J. Redox-dependent oligomerization through a leucine zipper motif is essential for MG53-mediated cell membrane repair. Am J Physiol, Cell Physiol. 2011;301(1):C106-C114.

28. Ma LL, et al. Hypercholesterolemia blocked sevoflurane-induced cardioprotection against ischemia-reperfusion injury by alteration of the MG53/RISK/GSK3 $\beta$ signaling. Int J Cardiol. 2013;168(4):3671-3678.

29. Kohr MJ, Evangelista AM, Ferlito M, Steenbergen C, Murphy E. S-nitrosylation of TRIM72 at cysteine 144 is critical for protection against oxidation-induced protein degradation and cell death. J Mol Cell Cardiol. 2014;69:67-74.

30. Murphy E, Kohr M, Sun J, Nguyen T, Steenbergen C. S-nitrosylation: a radical way to protect the heart. J Mol Cell Cardiol. 2012;52(3):568-577.

31. Cao CM, et al. MG53 constitutes a primary determinant of cardiac ischemic preconditioning. Circulation. 2010;121(23):2565-2574.

32. Zhang Y, et al. MG53 participates in ischaemic postconditioning through the RISK signalling pathway. Cardiovasc Res. 2011;91(1):108-115

33. Alloush J, Weisleder N. TRIM proteins in therapeutic membrane repair of muscular dystrophy. JAMA Neurol. 2013;70(7):928-931.

34. Liu J, et al. Cardioprotection of recombinant human MG53 protein in a porcine model of ischemia and reperfusion injury. J Mol Cell Cardiol. 2015;80:10-19.

35. Zhu H, et al. Amelioration of ischemia-reperfusion-induced muscle injury by the recombinant human MG53 protein. Muscle Nerve. 2015;52(5):852-858.

36. Jacobson MK, Payne DM, Alvarez-Gonzalez R, Juarez-Salinas H, Sims JL, Jacobson EL. Determination of in vivo levels of polymeric and monomeric ADP-ribose by fluorescence methods. Meth Enzymol. 1984;106:483-494.

37. McNeil PL. Cellular and molecular adaptations to injurious mechanical stress. Trends Cell Biol. 1993;3(9):302-307.

38. Corrado D, Basso C, Thiene G. Letter by Corrado et al regarding article, "sudden deaths in young competitive athletes: analysis of 1866 deaths in the United States, 1980-2006”. Circulation. 2009;120(16):e143.

39. Hagiwara N, Klewer SE, Samson RA, Erickson DT, Lyon MF, Brilliant MH. Sox6 is a candidate gene for p100H myopathy, heart block, and sudden neonatal death. Proc Natl Acad Sci USA. 2000;97(8):4180-4185.

40. Cai C, et al. MG53 regulates membrane budding and exocytosis in muscle cells. J Biol Chem. 2009;284(5):3314-3322.

41. Wittig I, Braun HP, Schägger H. Blue native PAGE. Nat Protoc. 2006;1(1):418-428. 
42. Phillips D, et al. Regulation of oxidative phosphorylation complex activity: effects of tissue-specific metabolic stress within an allometric series and acute changes in workload. Am J Physiol Regul Integr Comp Physiol. 2012;302(9):R1034-R1048.

43. Mertins P, et al. Proteogenomics connects somatic mutations to signalling in breast cancer. Nature. 2016;534(7605):55-62.

44. Chung YW, et al. Targeted disruption of PDE3B, but not PDE3A, protects murine heart from ischemia/reperfusion injury. Proc Natl Acad Sci USA. 2015;112(17):E2253-E2262.

45. Hausenloy DJ, Yellon DM. Cell membrane repair as a mechanism for ischemic preconditioning? Circulation 2010;121(23):2547-2549.

46. Murry CE, Wiseman RW, Schwartz SM, Hauschka SD. Skeletal myoblast transplantation for repair of myocardial necrosis J Clin Invest. 1996;98(11):2512-2523.

47. Corda D, Di Girolamo M. Mono-ADP-ribosylation: a tool for modulating immune response and cell signaling. Sci STKE. 2002;2002(163):pe53.

48. Adriouch S, Hubert S, Pechberty S, Koch-Nolte F, Haag F, Seman M. NAD+ released during inflammation participates in T cell homeostasis by inducing ART2-mediated death of naive T cells in vivo. J Immunol. 2007;179(1):186-194.

49. Zolkiewska A. Ecto-ADP-ribose transferases: cell-surface response to local tissue injury. Physiology (Bethesda). 2005;20:374-381.

50. Stevens LA, et al. Nonenzymatic conversion of ADP-ribosylated arginines to ornithine alters the biological activities of human neutrophil peptide-1. J Immunol. 2014;193(12):6144-6151.

51. Kato J, Zhu J, Liu C, Moss J. Enhanced sensitivity to cholera toxin in ADP-ribosylarginine hydrolase-deficient mice. Mol Cell Biol. 2007;27(15):5534-5543.

52. Rush J, et al. Immunoaffinity profiling of tyrosine phosphorylation in cancer cells. Nat Biotechnol. 2005;23(1):94-101.

53. Nagre N, et al. TRIM72 modulates caveolar endocytosis in repair of lung cells. Am J Physiol Lung Cell Mol Physiol. 2016;310(5):L452-L464.

54. Clarke MS, Caldwell RW, Chiao H, Miyake K, McNeil PL. Contraction-induced cell wounding and release of fibroblast growth factor in heart. Circ Res. 1995;76(6):927-934.

55. Bourgeois C, Okazaki I, Cavanaugh E, Nightingale M, Moss J. Identification of regulatory domains in ADP-ribosyltransferase-1 that determine transferase and NAD glycohydrolase activities. J Biol Chem. 2003;278(29):26351-26355.

56. Stevens LA, Levine RL, Gochuico BR, Moss J. ADP-ribosylation of human defensin HNP-1 results in the replacement of the modified arginine with the noncoded amino acid ornithine. Proc Natl Acad Sci USA. 2009;106(47):19796-19800. 